\title{
QUADRoTOR ATTITUdE STABILIZATION USING TAKAGI-SUGENOMODEL
}

\author{
Fouad Yacef ${ }^{1}$, Hana Boudjedir $^{1}$, Omar Bouhali $^{1}$, andHicham Khebbache ${ }^{2}$ \\ ${ }^{1}$ Automatic Laboratory of Jijel (LAJ), Automatic Control Department, Jijel University, \\ ALGERIA \\ yaceffouad@yahoo.fr, hana_boudjedir@yahoo.fr, bouhali_omar@yahoo.fr \\ ${ }^{2}$ Automatic Laboratory of Setif (LAS), Electrical Engineering Department, Setif \\ University, ALGERIA \\ khebbachehicham@yahoo.fr
}

\begin{abstract}
In this paper a robust controller for attitude stabilization of a Quadrotor UAV is proposed. For this we design a Takagi-Sugeno (T-S) model for Quadrotor modelling, and then we use Linear Matrix Inequality (LMI), and PDC (Parallel Disturbance Compensation) technique to design a nonlinear state feedback controller with pole placement in a pre-specified region of the operating space.The stabilityof the whole closed-loop system is investigated using quadratic Lyapunovfunction.To demonstrateits usefulness, the proposed design methodology is applied to the problem ofQuadrotor attitude stabilization. Simulation results show that the proposedLMI-based design methodology yields good transient performance.In addition, it is observed that the proposed state feedback controller provides superior stability robustness againstparameter variationsand measurement noise.
\end{abstract}

\section{KEYWORDS}

Linear Matrix Inequality (LMI), measurement noise,Parameter Variations,Parallel Disturbance Compensation (PDC), Pole Placement, Quadrotor UAV, Takagi-Sugenomodel.

\section{INTRODUCTION}

Unmanned Aerial Vehicles (UAVs) have been designed inthe military field since more than one half century. The mainobjective was to replace human pilot in a painful tasks andwhen the environment became hostile where the security ofpilots is not assured.These firsts designed UAV's date from the Second WorldWar; they have the dynamics and dimensions of plans and flew at very high altitudes [1].Quadrotor Helicopter is considered as one of the most popular UAV platform. This kind of helicopters are dynamically unstable, and therefore suitable control methods was used to make them stable, as back-stepping and sliding-mode techniques [2] [3].

In everyday life, the strategy how to solve a complex problem is called divide \& conquer. The problem is divided into simpler parts, which are solved independently and together yields the solution to the whole problem. The same strategy can be used for modelling and control of nonlinear systems, where the non-linear plant is substituted by locally valid set of linear sub models [4].The issue of stability and the synthesis of controllers for nonlinear systems described by continuous-time Takagi-Sugeno (T-S) models [5] have been considered actively. There has been also an increasing interest in the multiple model approach [6] [7] which also use the T-S systems to modelling.

DOI : $10.5121 /$ ijics.2012.2302 
During the last years, many works have been carried out to investigate the stability analysis and the design of state feedback controller of T-S systems. Using a quadratic Lyapunov function and Parallel Disturbance Compensation (PDC) technique, sufficient conditions for the stability and stabilisability have been established [8] [9]. The stability depends on the existence of a common positive definite matrix guarantying the stability of all local subsystems. The PDC control is a nonlinear state feedback controller. The gain of this controller can be expressed as the solution of a linear matrix inequality (LMIs) set [10].

\section{QUADROTOR DYNAMICAL MODEL}

We can describe the vehicle as having four propellers in cross configuration. The two pairs of propellers $(1,3)$ and $(2,4)$ turn in opposite directions. By varying the rotor speeds, one can change the lift forces and create motion. Thus, increasing or decreasing the four propeller's speeds together generates vertical motion. Changing the 2 and 4 propeller's speed conversely produces roll rotation coupled with lateral motion. Pitch rotation and the corresponding lateral motion result from 1 and 3 propeller's speed conversely modified as described in Figure 1. Yaw rotation is more subtle, as it results from the difference in the counter-torque between each pair of propellers.

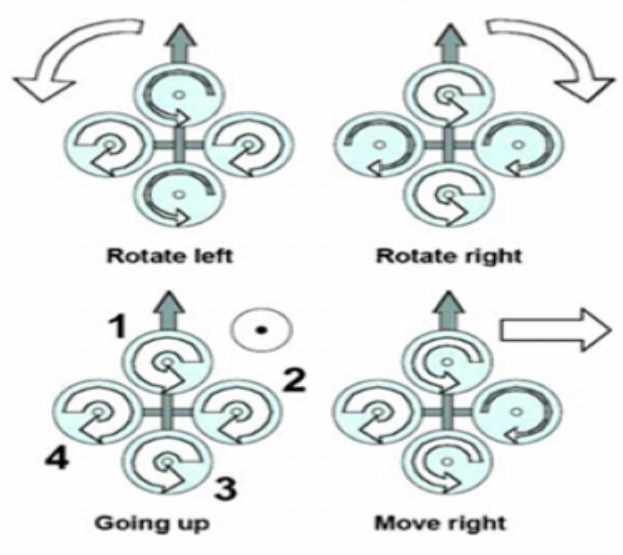

Figure 1.Quadrotor concept motion description

Quadrotor helicopter is one of the most complex flying systems that exist. This is due partly to the number of physical effects (Aerodynamic effects, gravity, gyroscopic, friction and inertial counter torques) acting on the system.

The first step before the control development is an adequate dynamic system modelling, especially for lightweight flying systems. Let us consider earth fixed frame $R^{b}$ and body fixed frame $R^{m}$, as seen in Figure 2. 


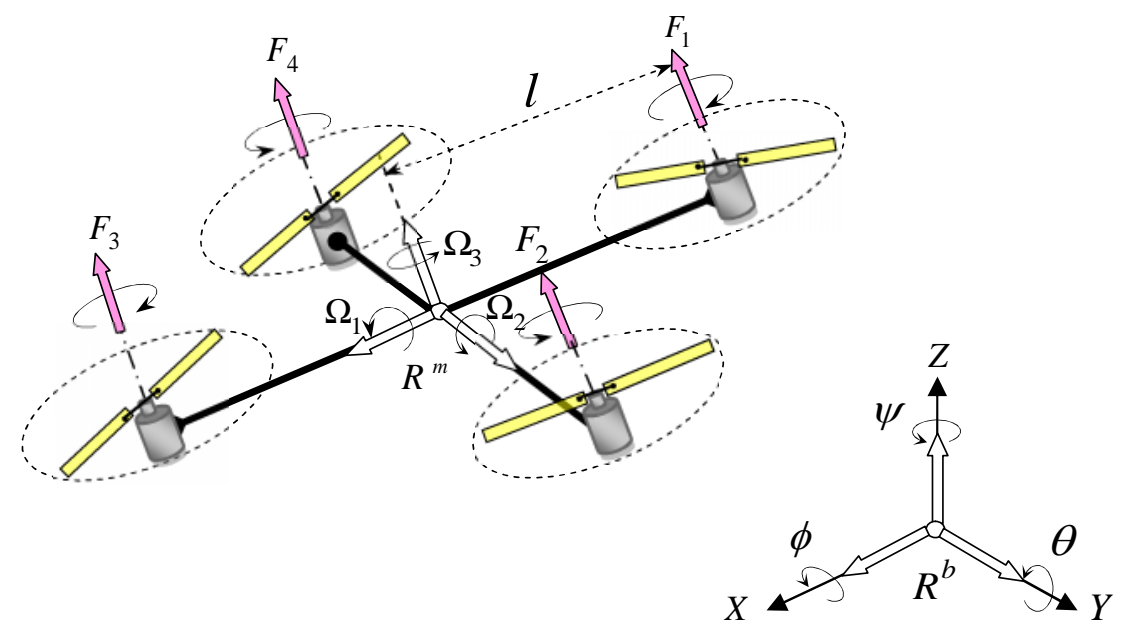

Figure 2.Quadrotor Architecture

The dynamics of the Quadrotor is described in the space bysix degrees of freedom according to the fixed inertial framerelated to the ground. This dynamics is related to thetranslational positions $(x, y, z)$ and the attitude described bythe Euler angles $(\phi, \theta, \psi)$. These six coordinates are theabsolute position of the centre of masse. The Euler angles are defined as follows:

- Roll angle $\phi:-\pi^{\prime} 2 \leq \phi \leq \pi^{\prime} 2$;

- Pitch angle $\theta:-\pi^{\prime} 2 \leq \theta \leq \pi^{\prime} 2$;

- Yaw angle $\psi:-\pi \leq \psi \leq \pi$.

The rotation transformation matrix $R$ from the inertial fixedframe $R^{b}$ to the body fixed frame $R^{m_{\text {is }}}$ given by:

$$
R=\left[\begin{array}{ccc}
c \psi c \theta & s \phi s \theta c \psi-s \psi c \phi & c \phi s \theta c \psi+s \psi s \phi \\
s \psi c \theta & s \phi s \theta s \psi+c \psi c \theta & c \phi s \theta s \psi-s \phi c \psi \\
-s \theta & s \phi c \theta & c \phi c \theta
\end{array}\right]
$$

With $s($.$) and c($.$) represent \sin ($.$) and \cos ($.$) respectively.$

To derive the dynamic model of the Quadrotor, the Newton Euler formalism will be used on both translation and rotation motions.In this work we mainly focus our interest to the attitude dynamics and we consider the reduced dynamical model as follows [11]:

$$
\left\{\begin{array}{l}
\ddot{\phi}=\dot{\theta} \dot{\psi} \frac{\left(I_{y}-I_{z}\right)}{I_{x}}-\frac{I_{r}}{I_{x}} \Omega_{r} \dot{\theta}-\frac{K_{f a x}}{I_{x}} \dot{\phi}^{2}+\frac{1}{I_{x}} u_{1} \\
\ddot{\theta}=\dot{\phi} \dot{\psi} \frac{\left(I_{z}-I_{x}\right)}{I_{y}}+\frac{I_{r}}{I_{y}} \Omega_{r} \dot{\phi}-\frac{K_{f a y}}{I_{y}} \dot{\theta}^{2}+\frac{1}{I_{y}} u_{2} \\
\ddot{\psi}=\dot{\theta} \dot{\phi} \frac{\left(I_{x}-I_{y}\right)}{I_{z}}-\frac{K_{f a z}}{I_{z}} \dot{\psi}^{2}+\frac{1}{I_{z}} u_{3}
\end{array}\right.
$$


The inputs of the system are $u_{1}, u_{2}, u_{3}$ and $\Omega_{r}$ as a disturbance, obtaining:

$$
\left\{\begin{array}{l}
u_{1}=b l\left(\omega_{4}^{2}-\omega_{2}^{2}\right) \\
u_{2}=b l\left(\omega_{3}^{2}-\omega_{1}^{2}\right) \\
u_{3}=d\left(\omega_{1}^{2}-\omega_{2}^{2}+\omega_{3}^{2}-\omega_{4}^{2}\right) \\
\Omega_{r}=\omega_{1}-\omega_{2}+\omega_{3}-\omega_{4}
\end{array}\right.
$$

\section{QUADROTOR TAKAGI-SUGENO MODEL}

\subsection{Takagi-Sugeno model}

A T-S model is based on the interpolation between several LTI (linear time invariant) local models as follow:

$$
\dot{x}_{m}(t)=\sum_{i=1}^{r} \mu_{i}(\xi(t))\left(A_{i} x_{i}(t)+B_{i} u(t)\right)
$$

Where $r$ is the number of sub-models, $x_{m}(t) \in \mathbb{R}^{p}$ is the state vector, $u(t) \in \mathbb{R}^{h}$ is the input vector $A_{i} \in \mathbb{R}^{p \times p}, B_{i} \in \mathbb{R}^{p \times h}$,and $\xi(t) \in \mathbb{R}^{q}$ is the decision variable vector.

The variable $\xi(t)$ may represent measurable states and/or inputs and the form of this variable may leads to different class of systems: if $\xi(t)$ is known functions than the model (4) represents a nonlinear system and if there are unknown we consider that this leads to linear differential inclusion (LDI). This variable can also be a function of the measurable outputs of the system. The normalized activation function $\mu_{i}(\xi(t))$ in relation with the ith sub-model is such that:

$$
\left\{\begin{array}{l}
\sum_{i=1}^{r} \mu_{i}(\xi(t))=1 \\
0 \leq \mu_{i}(\xi(t)) \leq 1
\end{array}\right.
$$

According to the zone where evolves the system, this function indicates the more or less important contribution of the local model corresponding in the global model (T-S model).

The global output of T-S model is interpolated as follows:

$$
y_{m}(t)=\sum_{i=1}^{r} \mu_{i}(\xi(t))\left(C_{i} x_{i}(t)+D_{i} u(t)\right)
$$

Where $y_{m}(t) \in \mathbb{R}^{l}$ is the output vector and $C_{i} \in \mathbb{R}^{l \times p}, D_{i} \in \mathbb{R}^{l \times h}$. More detail about this type of representation can be found in [5].

\subsection{Quadrotor Takagi-Sugeno model}

The behaviour of a nonlinear system near an operating point $\left(x_{i}, u_{i}\right)$, can be described by a linear time-invariant system (LTI). Using Taylor series about $\left(x_{i}, u_{i}\right)$ and keeping only the linear terms yields: 


$$
\dot{x}(t)=A_{i}\left(x(t)-x_{i}\right)+B_{i}\left(u(t)-u_{i}\right)+f\left(x_{i}, u_{i}\right)
$$

Which can written as

$$
\dot{x}(t)=A_{i} x(t)+B_{i} u(t)+d_{i}
$$

With:

$$
A_{i}=\left.\frac{\partial f(x, u)}{\partial x}\right|_{\substack{x=x_{i} \\ u=u_{i}}}, B_{i}=\left.\frac{\partial f(x, u)}{\partial u}\right|_{\substack{x=x_{i} \\ u=u_{i}}}, f(x, u)=\dot{x}(t), d_{i}=f\left(x_{i}, u_{i}\right)-A_{i} x_{i}-B_{i} u_{i}
$$

After calculation we obtained:

$$
A_{i}=\left[\begin{array}{cccccc}
0 & 1 & 0 & 0 & 0 & 0 \\
0 & a_{1} & 0 & a_{2} \dot{\psi}+a_{3} & 0 & a_{2} \dot{\theta} \\
0 & 0 & 0 & 1 & 0 & 0 \\
0 & a_{5}+a_{4} \dot{\psi} & 0 & a_{6} & 0 & a_{4} \dot{\phi} \\
0 & 0 & 0 & 0 & 0 & 1 \\
0 & a_{7} \dot{\theta} & 0 & a_{7} \dot{\phi} & 0 & a_{8}
\end{array}\right], \quad B_{i}=\left[\begin{array}{ccc}
0 & 0 & 0 \\
b_{1} & b_{2} & b_{3} \\
0 & 0 & 0 \\
b_{4} & b_{5} & b_{6} \\
0 & 0 & 0 \\
0 & 0 & b_{7}
\end{array}\right]
$$

Combined local affine models (8)using Gaussian activation function we describe the dynamic model of the Quadrotor by a T-S model:

$$
\left\{\begin{array}{l}
x_{m}(t)=\sum_{i=1}^{3} \mu_{i}(\xi(t))\left(A_{i} x_{m}(t)+B_{i} u(t)+d_{i}\right) \\
y_{m}(t)=C x_{m}(t)
\end{array}\right.
$$

With:

$$
\mu_{i}(\xi(t))=\frac{\omega_{i}(\xi(t))}{\sum_{j=1}^{3} \omega_{i}(\xi(t))}, \omega_{i}(\xi(t))=\prod_{j=1}^{3} \exp \left(-\frac{\left(\xi_{j}(t)-c_{i, j}\right)^{2}}{2 \sigma_{i, j}^{2}}\right)
$$

-The vector of decision variables $\xi(t)=\left[\begin{array}{lll}\dot{\phi} & \dot{\theta} & \dot{\psi}\end{array}\right]^{T}$

- The parameters of activations functions $\left(c_{i, j}, \sigma_{i, j}\right)$ are given as:

- The centres $c_{i, j}$ are defined according to the operation point.

- The Dispersions $\sigma_{i, j}$ are defined by optimization of a criterion, which represent the quadratic error between Takagi-Sugeno model outputs and nonlinear system outputs, using Particle Swarm Optimisation algorithm(PSO) [12][13].

-The operating points are chosen to cover maximum space of the operating space, with small number of local models. The attitude of Quadrotor (roll, pitch, and yaw) has a limited bound 
$\left(-\pi^{\prime} 2 \leq \phi \leq \pi^{\prime} 2,-\pi^{\prime} 2 \leq \theta \leq \pi^{\prime} 2,-\pi \leq \psi \leq \pi\right)$, for this reason we use three local models to cover this space.Linear local model are defined in this table as follow:

Table 1. Operation Points Parameters.

\begin{tabular}{|c|c|cccc|}
\hline $\mathbf{N}^{\circ} \mathbf{O . P}$ & Parameters & \multicolumn{5}{|c|}{$\boldsymbol{d}_{\boldsymbol{i}}$} \\
\hline 1 & $\dot{\phi}=\dot{\theta}=\dot{\psi}=-0.523 \mathrm{rad} / \mathrm{s}$ & {$\left[\begin{array}{llllll}0 & 0.1964 & 0 & -0.1964 & 0 & 0\end{array}\right]^{T}$} \\
\hline 2 & $\dot{\phi}=\dot{\theta}=\dot{\psi}=0 \mathrm{rad} / \mathrm{s}$ & {$\left[\begin{array}{llllll}0 & 0 & 0 & 0 & 0 & 0\end{array}\right]^{T}$} & \\
\hline 3 & $\dot{\phi}=\dot{\theta}=\dot{\psi}=0.523 \mathrm{rad} / \mathrm{s}$ & {$\left[\begin{array}{llllll}0 & 0.2771 & 0 & -0.2771 & 0 & 0\end{array}\right]^{T}$} \\
\hline
\end{tabular}

\subsection{Quadrotor Takagi-Sugeno model validation}

The input signals (rotors velocities) most appropriated for the local models network validation, and exit all dynamic of the system in this case is the Pseudo-Random Binary Signal (SBPA) due to different causes:the SBPA signal has a null mean and a variance that close to one, which allows the excitation of very good frequency range (dynamics system) without moving away too much the system from the operating point. It is periodic deterministic signal white-noise-like properties very adapted for identification and validation tasks.

A typical value of the amplitude of the SBPAis from $0.5 \%$ to $5 \%$ from the value of the operating point to which the SBPA is applied, in this case the amplitude of the SBPA is given as

$$
A_{S B P A}=\omega_{e q} \pm 0.005 * \omega_{e q}, \quad \omega_{e q}=\sqrt{\frac{m g}{4 b}} .
$$

To validate the synthesized Takagi-Sugeno model a SBPA (input signal) is used, for Quadrotor nonlinear system and the T-S model. We simulate the two systems in parallel and we compare the resulting curves.

Figure 3 present the input signals of Quadrotor, which are SBPA signals with variable amplitude. This SBPA excite all dynamic of the system.
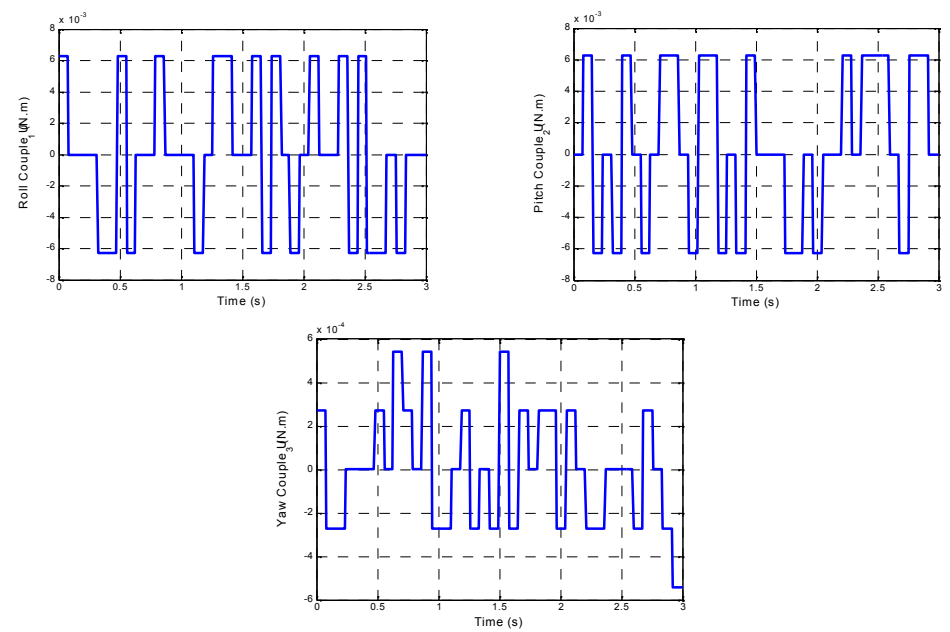

Figure 3. Validation input signals 
Figure 4 present the attitude of Quadrotor and corresponding output of T-S model. We show the resemblance between the output of T-S model and Quadrotor nonlinear system. These results prove the quality of the approximation of a nonlinear system by a T-S model.

Figure 5 present attitude acceleration errors, which are close to a white-noise with null mean and a variance that close to one. Saw the designing T-S model give good approximation of the Quadrotor nonlinear system for a specific region of the operating space.
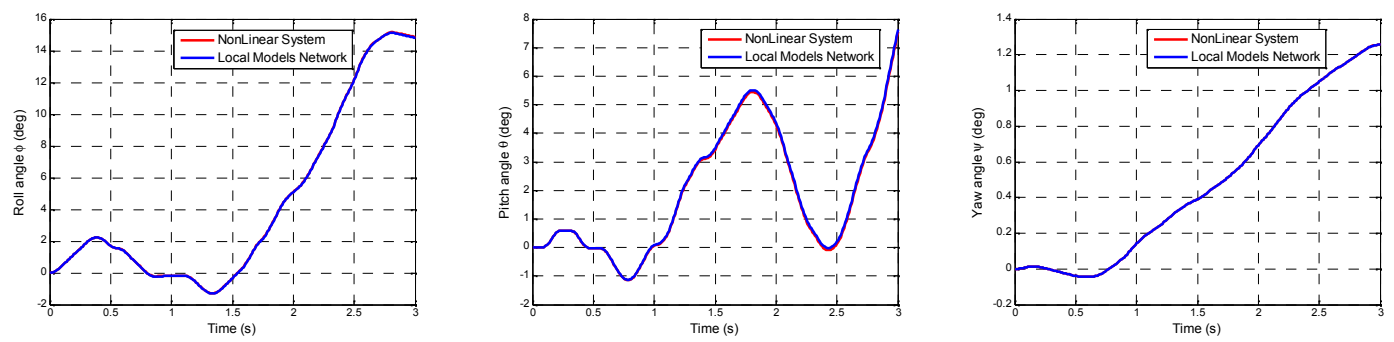

Figure 4. Takagi-Sugeno model and Quadrotor's outputs
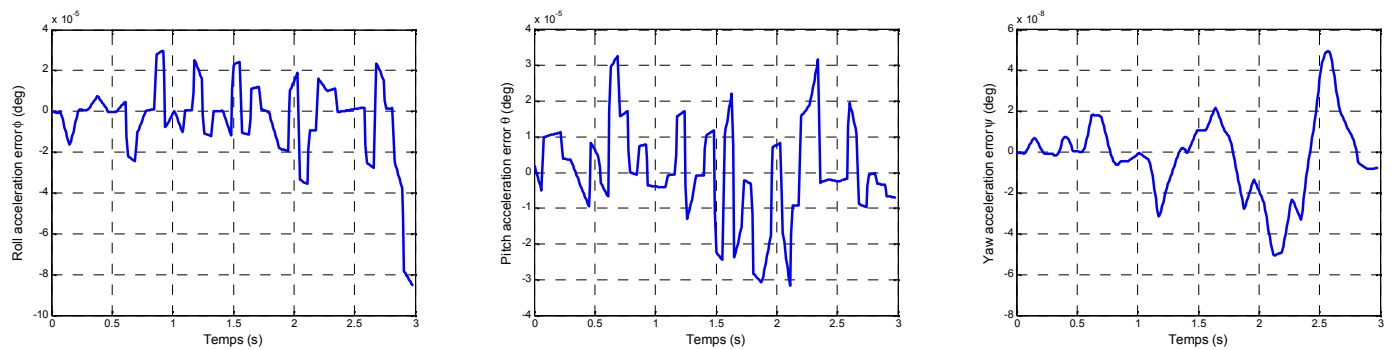

Figure 5. Attitude acceleration errors

\section{Controller Design}

\subsection{State feedback controller}

The concept of PDC, following the terminology [8], is utilized to design state-feedback controller on the basis of the T-S model (10). Linear control theory can be used to design the control law, because T-S model is described by linear state equations. The controller law is a convex linear combination of the local controller associated with the corresponding local sub-model. It can present as:

$$
U(t)=-\sum_{i=1}^{r} \mu_{i}(\xi(t)) u_{i}(t)=-\sum_{i=1}^{r} \mu_{i}(\xi(t)) K_{i} x(t)
$$

With: $K_{i}$ is $r$ vector of feedback gains.

It should be noted that the designed controller shares the same models sets with T-S models, and resulting controller (11) is nonlinear in general since the coefficient of the controller depends nonlinearly on the system input and output via the weighting functions. Substituting (11) into (10), the closed-loop T-S model can be represented by: 


$$
\dot{x}(t)=\sum_{i=1}^{r} \sum_{j=1}^{r} \mu_{i}(\xi(t)) \mu_{j}(\xi(t))\left(A_{i}-B_{i} K_{j}\right) x(t)
$$

The constant $d_{i}$ was neglected in this formulate, because the control law can compensate the effect of this bias term.

\subsection{Stabilisation using PDC}

A sufficient quadratic stability condition derived by Tanaka and Sugeno[14] for ensuring stability of (12) is given as follows:

Theorem 1: The closed-loop T-S model (12) is quadratic-ally stable for some stable feedback $K_{i}$ (via PDC scheme) if there exists a common positive definite matrix $P$ such that:

$$
\begin{gathered}
G_{i i}^{T} P+P G_{i i}<0 \quad \forall i \in I_{r} \\
\left(\frac{G_{i j}+G_{j i}}{2}\right)^{T} P+P\left(\frac{G_{i j}+G_{j i}}{2}\right)<0, \quad \forall(i, j) \in I_{r}^{2}, i<j
\end{gathered}
$$

With: $G_{i i}=A_{i}-B_{i} K_{j}, \mu_{i}(\xi(t)) \mu_{j}(\xi(t)) \neq 0$.

Which is an LMI in $P$ when $K_{i}$ are predetermined.However, our objective is to design the gain matrix $K_{i}$ such that conditions (13) are satisfied. That is, $K_{i}$ are not pre-determined matrices any longer, but matrix variables.This is the quadratic stability problem and can be recast as an LMI feasibility problem. With linear fractional transformation $X=P^{-1}$ and $N_{i}=K_{i} X$, we may rewrite (13) as an LMI problem in $N_{i}, X$ and $S_{i j}[15]$ :

$$
\begin{gathered}
X>0 \\
X A_{i}{ }^{T}+A_{i} X-N_{i}^{T} B_{i}^{T}-B_{i} N_{i}+S_{i i}<0, \quad \forall i \in I_{r} \\
X A_{i}{ }^{T}+A_{i} X+X A_{j}{ }^{T}+A_{j} X-N_{j}^{T} B_{i}^{T}-B_{i} N_{j} \\
-N_{i}{ }_{i} B_{j}^{T}-B_{j} N_{i}+2 S_{i j} \leq 0, \quad \forall(i, j) \in I_{r}^{2}, i<j \\
\left(\begin{array}{ccc}
S_{11} & \cdots & S_{1 n} \\
\vdots & \ddots & \vdots \\
S_{1 n} & \cdots & S_{n n}
\end{array}\right)>0
\end{gathered}
$$

With: $S_{i j}=X Q_{i j} X, \forall i \in\{1, \ldots, r\}, Q_{i j}$ are symmetric matrix.

\subsection{LMI formulation for Pole placement}

In order to achieve some desired transientperformance, a pole placement should beconsidered. For many problems, exact poleassignment may not be necessary; it suffices tolocate the pole of the closed loop system in a sub-regionof the complex left half plane. This sectiondiscusses a pole assignment in LMI regions. For this purpose, we introduce the following LMI-based representation of stability regions [16] [17].

Definition:A subset $D$ of the complex planeis called an LMI region if there exist a symmetricmatrix $\alpha=\left(\alpha_{i j}\right) \in \mathbb{R}^{p \cdot p}$ and a matrix $\beta=\left(\beta_{i j}\right) \in \mathbb{R}^{p \cdot p}$ such that: 


$$
D=\left\{z \in \sqcup: f_{D}(z)<0\right\}
$$

Where: $f_{D}(z)=\left(\alpha_{i j}+\beta_{i j} z+\beta_{i j} \bar{z}\right), \forall i, j \epsilon\{1, \ldots, p\}$

Theorem 2:A matrix $A$ is D-stable if and onlyif there exists a symmetric positive definite matrix $X$ such that:

$$
M_{D}(X, A)<0
$$

Where: $M_{D}(X, A)=\alpha \otimes X+\beta \otimes(A X)+\beta^{T} \otimes(A X)^{T}$

For example, a circle region $D$ centred at $(-q, 0)$ with radius $\rho>0$ can be obtained by taking thematrices and as follows:

$$
\alpha=\left(\begin{array}{cc}
-\rho & q \\
q & -\rho
\end{array}\right) \text {, and } \beta=\left(\begin{array}{ll}
0 & 1 \\
0 & 0
\end{array}\right)
$$

What makes it possible to obtain the expression ofthe characteristic function:

$$
f_{D}(z)=\left(\begin{array}{cc}
-\rho & z^{*}+q \\
z+q & -\rho
\end{array}\right)
$$

As it is shown in figure 6, this region which includecircular region, allows fixing a lower bound on boththe exponential decay rate: $\rho-q$ and the dampingratio: $\zeta_{\min }=\sqrt{1-\left(\rho^{2 /} q^{2}\right)}(\rho<q)$ of the closed-loopresponse, and thus is very common in practical control design.

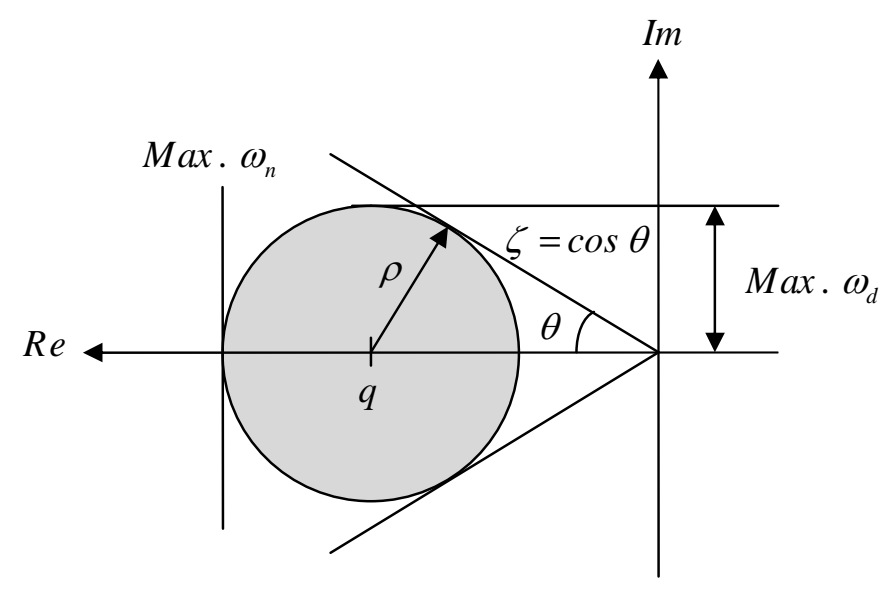

Figure 6. Circular region $(D)$ for pole location

Since the prescribed LMI region (17) will be addedas supplementary constraints to these of thetheorem 1, it should be noted that itonly suffices to locate the poles of the dominantterm in the prescribe LMI regions, i.e. the case of $i=j$. It follows that the closed loop T-S model (12) is Dstable if there exists asymmetric matrix $X$ such that [18]: 
International Journal of Instrumentation and Control Systems (IJICS) Vol.2, No.3, July 2012

$$
\left(\begin{array}{cc}
-\rho X & q X+X\left(A_{i}+B_{i} K_{j}\right)^{T} \\
q X+\left(A_{i}+B_{i} K_{j}\right) X & -\rho X
\end{array}\right)
$$

With the same change of variables $N_{i}=K_{i} X$ leads to thefollowing LMI formulation:

$$
\left(\begin{array}{cc}
-\rho X & q X+X A_{i}^{T}+B_{i}^{T} N_{i}^{T} \\
q X+X A+B_{i} N_{i} & -\rho X
\end{array}\right), i=j
$$

By combining Theorems 1 and 2 leads to the following LMI formulation of two objectives statefeedback synthesis problem, such that the resulting controller meets both the global stability and the desired transient performance simultaneously. The closed loop T-S model (12) is stabilizable in the specified region $D$ ifthere exists asymmetric matrix $X$ such that [16]:

$$
\begin{gathered}
X>0 \\
X A_{i}^{T}+A_{i} X-N_{i}^{T} B_{i}^{T}-B_{i} N_{i}+S_{i i}<0, \quad \forall i \in I_{r} \\
X A_{i}^{T}+A_{i} X+X A_{j}{ }^{T}+A_{j} X-N_{j}^{T} B_{i}^{T}-B_{i} N_{j} \\
-N_{i}^{T} B_{j}^{T}-B_{j} N_{i}+2 S_{i j} \leq 0, \quad \forall(i, j) \in I_{r}^{2}, i<j \\
\left.-\rho X \quad \begin{array}{cc}
- & q X+X A_{i}^{T}+N_{i}^{T} B_{i}^{T} \\
q X+A_{i} X+B_{i} N_{i} & -\rho X
\end{array}\right)<0, \quad \forall i \in I_{r} \\
\left(\begin{array}{ccc}
S_{11} & \cdots & S_{1 n} \\
\vdots & \ddots & \vdots \\
S_{1 n} & \cdots & S_{n n}
\end{array}\right)>0
\end{gathered}
$$

With: $S_{i j}=X Q_{i j} X, K_{i}=N_{i} X, \forall i \in\{1, \ldots, r\}$

\subsection{State feedback gains calculation}

Using Theorem 1 and 2; can design a nonlinear state feedback controller that guarantees global stability while provides desired transient behaviour by constraint the closed-loop poles in $D$. The stability region $D$ is a circle of $\operatorname{centre}(q, 0)$ and $\operatorname{radius} \rho$ and the LMI synthesis is performed for a set of values $(q, \rho)=(4,1)$.

Then the LMI region has the following characteristic function:

$$
f_{D}(z)=\left(\begin{array}{cc}
-1 & z^{*}+4 \\
z+4 & -1
\end{array}\right)
$$

This circle region puts a lower bound on both exponential decay rate $q-\rho=3 \mathrm{rad}^{\prime}$ sand damping ratio $\zeta=\sqrt{1-\left(\rho^{2 /} q^{2}\right)}=0.97$ of the closed-loop response.By solving LMI feasibility problem (20), we can obtain a positive symmetric matrix $X$ (by interior-point method in Matlab LMI-toolbox), and stat feedback Matrix $K_{i}$. 


\subsection{Simulation results}

The controller described above was simulated for the nonlinear Quadrotor system. Simulations are made for initial values equal to $\left(\phi_{0}, \theta_{0}, \psi_{0}\right)=(20,40,60) \mathrm{deg}$ for roll angle, $(-20,-40,-60) \mathrm{deg}$ for pitch angle, and $(40,80,120) \mathrm{deg}$ for yaw angle, and equal to zeros for tracking simulation. The values of the model parameters used for simulations are the following:

$m=0.486 \mathrm{~kg}, l=0.225 \mathrm{~m}, g=9.81 \mathrm{~m} / \mathrm{s}^{2}, d=3.23 \times 10^{-7} \mathrm{~N} . \mathrm{m} /(\mathrm{rad} . \mathrm{s}), b=2.98 \times$ $10^{-5} \mathrm{~N}^{\prime}(\mathrm{rad} . \mathrm{s}), I_{x}=I_{y}=3.82 \times 10^{-3} \mathrm{~kg} \cdot \mathrm{m}^{2}, I_{z}=7.65 \times 10^{-3} \mathrm{~kg} \cdot \mathrm{m}^{2}, K_{\text {fax }}=K_{\text {fay }}=$ $5.567 \times 10^{-4}, K_{f a z}=6.354 \times 10^{-4}$.

The results of state feedback controller are shown in Figure 7 which indicates the output of Quadrotor nonlinear system (Quadrotor attitude, Roll, Pitch, and Yaw), and the corresponding control inputs in figure 8 .

From the resultsof figure 7,8 ; it can be noticed that state the feedback controller provides good transient performance(stabilization time, overtaking...), while, controller give stable response regardless of any initial displacement.The control inputs are smoother and realizable.
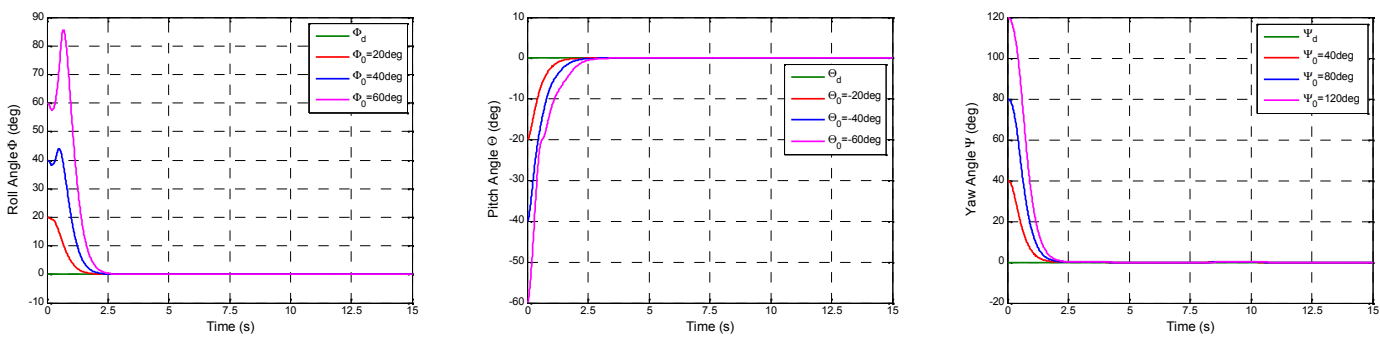

Figure 7. Quadrotor Attitude $(\phi, \theta, \psi)$ for state feedback controller
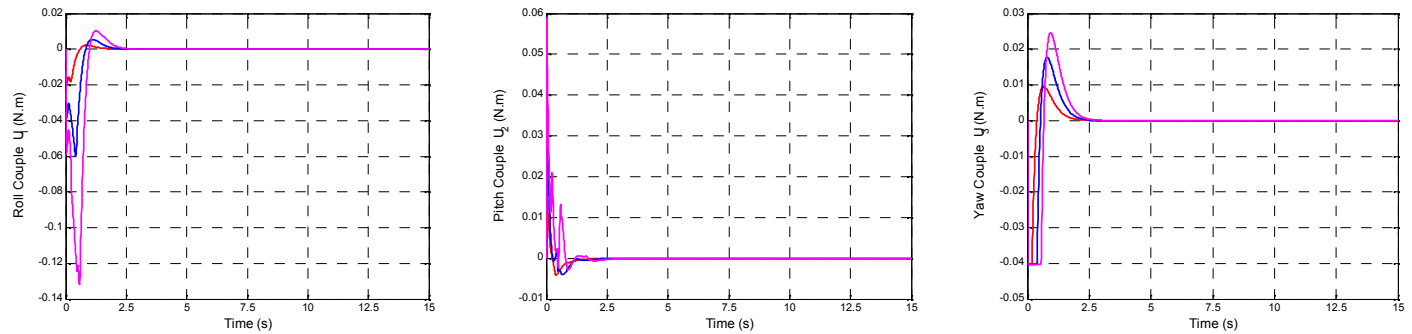

Figure 8. Quadrotor control inputs $\left(u_{1}, u_{2}, u_{3}\right)$ for state feedback controller

To check the robustness of the proposed controls two tests are used; the first again measurement noise, and the second: again parameter variations.

- Measurement noise of a normal distribution, a covariance equal to 1, a zero mean, and amplitude close to 0.05 are added to the measured variables as shown in figures 10, 11.

- A change of $100 \%$ for $\left(I_{x}, I_{y}, I_{z}\right)$ parameters and $40 \%$ variation of $b_{i}$ parameters are performed between 20 to 40 seconds (figures 12, 13).

Figure 10 represent measurement noisetest, for Quadrotor attitude tracking (Roll, Pitch, and Yaw), with a sinusoidal trajectory, we can clearly see a good tracking of desired trajectories, 
International Journal of Instrumentation and Control Systems (IJICS) Vol.2, No.3, July 2012

which confirms the robustness of the proposed controlleragainmeasures noise. Figure 11 represent inputs control system.

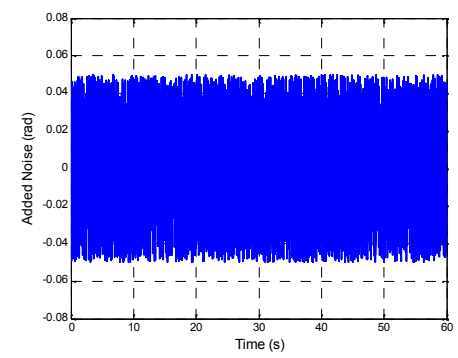

Figure 9. Measures noise added
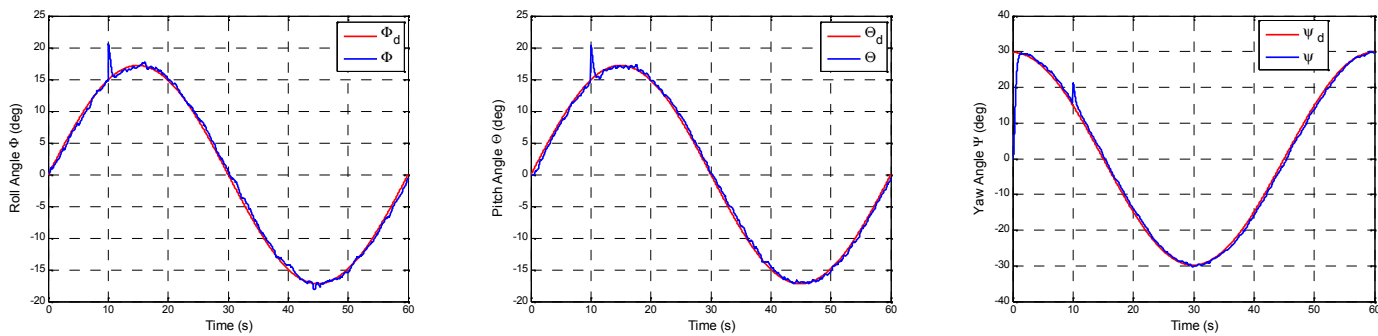

Figure 10. Quadrotor Attitude $(\phi, \theta, \psi)$ for measurement noise test
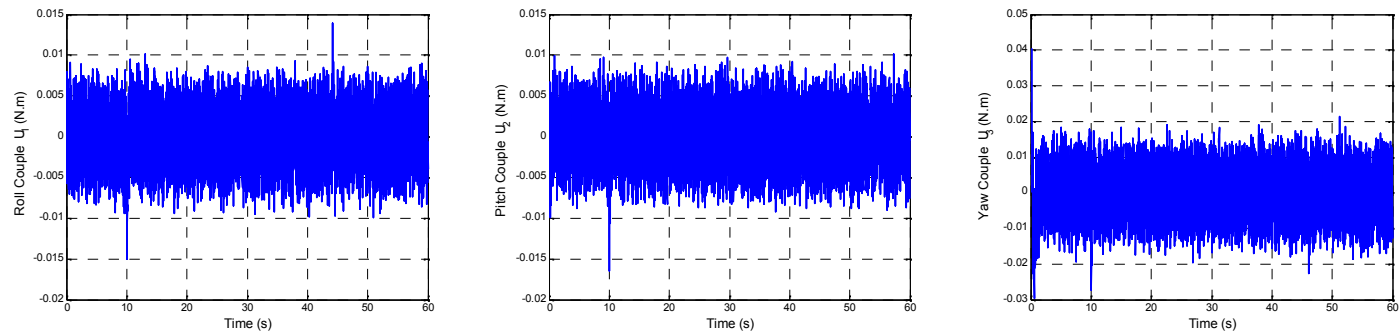

Figure 11. Quadrotor control inputs $\left(u_{1}, u_{2}, u_{3}\right)$ for measurement noise test
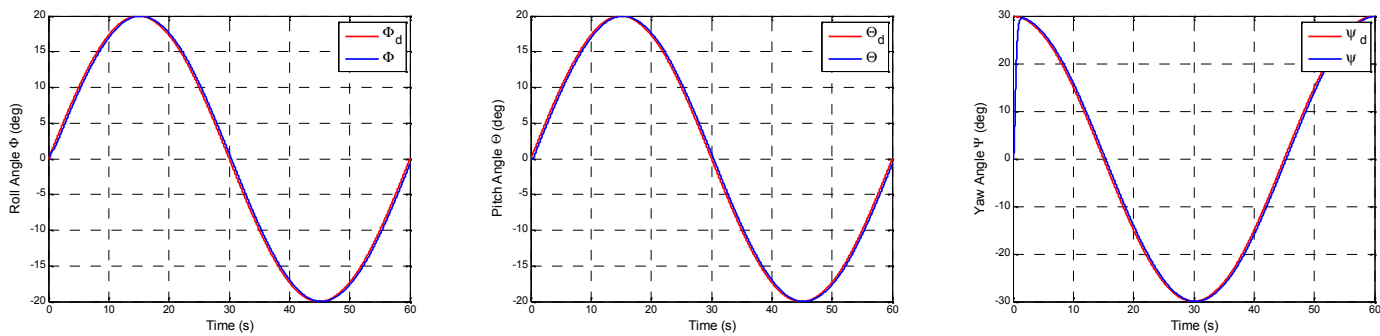

Figure 12. Quadrotor Attitude $(\phi, \theta, \psi)$ for parameter variation test 
International Journal of Instrumentation and Control Systems (IJICS) Vol.2, No.3, July 2012
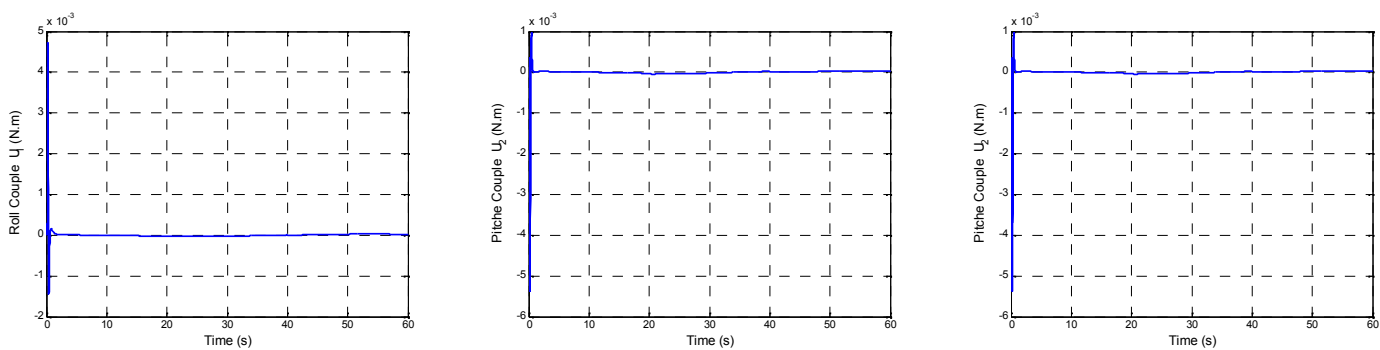

Figure 13. Quadrotor control inputs $\left(u_{1}, u_{2}, u_{3}\right)$ for parameter variation test

Figure 12 represent parameter variationstest, for Quadrotor attitude tracking (Roll, Pitch, and Yaw), with a sinusoidal trajectory, we can clearly see a good tracking of desired trajectories, which confirms the robustness of the proposed controlleragainparameter variation. Figure 13 represent inputs control system.

\section{Conclusions}

In this paperthe problem of Quadrotor attitude stabilization isresolved using T-S modeland state feedback controller. A T-S model is designed for Quadrotor modelling. We use a Quadratic Lyapunov function to prove the stability of closed loop system. The designed methodologyis based on ParallelDistributed Compensation technique and pole placement in LMI region.Simulation results showed that the proposedcontroller providesstable response regardless of any initial conditions.In addition, it is observed that theproposed state feedback controller providessuperior stability robustness against parameter variations and measurement noise.

\section{APPENDIX}

Parameters of $A_{i}$ and $B_{i}$ matrix

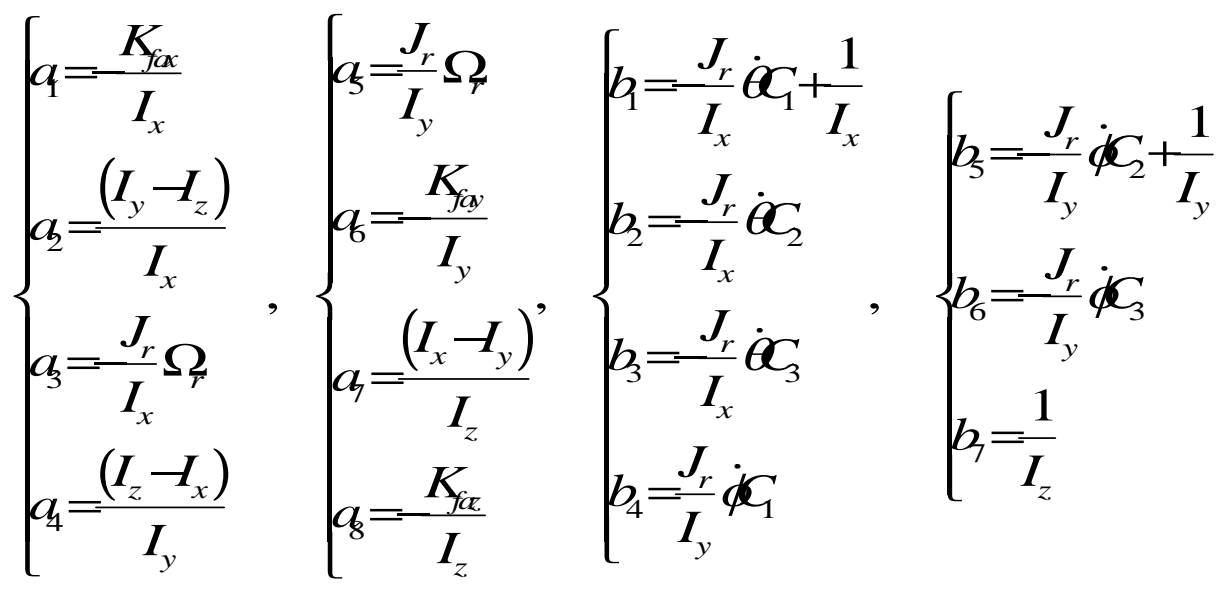


International Journal of Instrumentation and Control Systems (IJICS) Vol.2, No.3, July 2012

\section{REFERENCES}

[1] P. Brisset, (2004) "Drones civils Perspectives et réalités", Ecole Nationale del'Aviation Civile Aout 2004.

[2] T. Madani\& A. Benallegue, (2006) "Backstepping Sliding Mode Control Applied to a Miniature Quadrotor Flying Robot", IEEE Conference on Industrial Electronics, pp. 700-705.

[3] Y. Yu, J.Changhong, \& W.Haiwei, (2010) "Backstepping control of each channel for a Quadrotor aerial robot", International Conference on Computer, Macaronis, Control and Electronic Engineering (CMCE), pp. 403-407.

[4] J. Novák. (2007) "linear system identification and control using local model networks". Athesis submitted in fulfilment of the requirements for the $\mathrm{PhD}$ degree, Faculty of Applied Informatics, Tomas Bata University, Zlín, Czech.

[5] T. Takagi \& M. Sugeno, (1985) "Fuzzy identification of systems and its applications to model and control", IEEE Transactions on Systems, Man, and Cybernetics, vol. 15, pp. 116-132.

[6] M. Chadli, D. Maquin\& J.Ragot, (2001) "on the stability analysis of multiple models",in Proceeding of the ECC, Portugal, 2001, pp. 1894-1899.

[7] R. Murray-Smith \& T. A. Johansen, (1997) multiple model approaches to modelling and control, Taylor and Francis Publishers. France, 1997.

[8] H. O. Wang, K. Tanaka, \& M. Griffin, (1996) "An approache to fuzzy controle of non linear systems : stability and design issues," IEEE Transaction on fuzzy system, vol. 4, pp. 14-23.

[9] K. Tanaka, . T. Ikeda, \& Y. Y. He, (1998) "Fuzzy regulators and fuzzy observers : relaxed stability conditions and LMI-based design," IEEE Transaction on fuzzy system, vol. 6, pp. 250-256.

[10] S. Boyd, L. El Ghaoui, E. Feron, \& V. Balakrishnan (1994) "Linear Matrix Inequalities in System and Control Theory," SIAM, Philadelphia, USA.

[11] H .Bouadi, S. S. Cunha, A. Drouin,\& F. M. Camino,(2011) "Adaptive Sliding Mode Control for Quadrotor Attitude Stabilization and Altitude Tracking",IEEE International Symposium on Computational Intelligence and Informatics, pp. 449-455.S.

[12] F. Yacef\& F. Boudjema,(2011) "Local Model Network for non linear modelling and control of an UAV Quadrotor", International Conference on Automatic and Mechatronics (CIAM), November 2224, Oran, Algeria, pp. 247-252.

[13] F. Yacef, O. Bouhali, H. Khebbache\& F. Boudjema, (2012) "Takagi-Sugeno Model for Quadrotor Modelling and Control Using Nonlinear State Feedback Controller", International Journal of Control Theory and Computer Modelling (IJCTCM),Vol.2, No.3, pp. 9-24, May 2012.

[14] K. Tanaka \& M. Sugeno, (1992) "Stability analysis and design of fuzzy control systems", Fuzzy Set and Systems, vol. 45, pp. 135-156.

[15] M. Chadli, (2002)"Stability and control of multiple models:LMI approach", INPL thesis (in French), France.mchadli.voila.net/THESECHADLI.pdf.

[16] S. K. Hong \& Y. Nam, (2003) "Stable fuzzy control system design with pole-placement constraint: an LMI approach",Computers in Industry, vol. 51, pp. 1-11.

[17] M. Chadli, D. Maquin, J. Ragot, (2002) "Static Output Feedback for Takagi-SugenoSystems: an LMI Approach", Proceedings of the 10th Mediterranean Conference on Control and Automation,July 9-12, Lisbon, Portugal.

[18] M. Chilali\& P. Gahinet, (1996) "H $\infty$ Design with pole placement constraints: an LMI approach",IEEE Transaction on Automatic Control, vol. 41, pp. 358-367. 


\section{Authors}

FouadYacefis currently a Ph.D. student at the Automatic Control Department of JijelUniversity, Algeria. He received the Engineer degree in Automatic Control from JijelUniversity, Algeria in 2009, and Magister degree in Automatic Controlfrom the MilitaryPolytechnic School of Algeria(EMP), in November 2011.Since December 2011, he has been a researcher in Automatic Laboratory of Jijel (LAJ). His research interests include Aerialrobotics, linear and nonlinear control, analysis and design of intelligent control and renewable energy systems.

HanaBoudjedir received the Engineer degree and Magister degree in Automatic Control from Jijel University, Algeria in 2008, and 2010, respectively. She is currently a Ph.D. student at the Automatic Control Department of JijelUniversity, Algeria.Since December 2011, she has beena researcher in Automatic Laboratory of Jijel (LAJ). Her research interests includeaerialrobotics, nonlinear control systems, analysis and design of intelligent control systems.

Omar Bouhali He received the engineer degree and the master degree in automatic from national polytechnic school of Algeria, in 1996 and 1998 respectively. He received the Ph. D. degree from the Central School of Lille, France and National Polytechnic School of Algeria in 2007. Since 1998, he has been a lecturer in the automatic department of Jijel University, Algeria. And he is a researcher in Automatic Laboratory of Jijel (LAJ). He is working in the development of renewable energy based power systems with inertial storage using multilevel converters.

HichamKhebbacheis Graduate student (Magister) of Automatic Control at the Electrical Engineering Department of Setif University, ALGERIA. He received the Engineer degree in Automatic Control from Jijel University, ALGERIA in 2009. Now he is with AutomaticLaboratory of Setif (LAS). His research interests include Aerial robotics, Linear and Nonlinear control, Robust control, Fault tolerant control (FTC), Diagnosis, Fault detection and isolation(FDI).
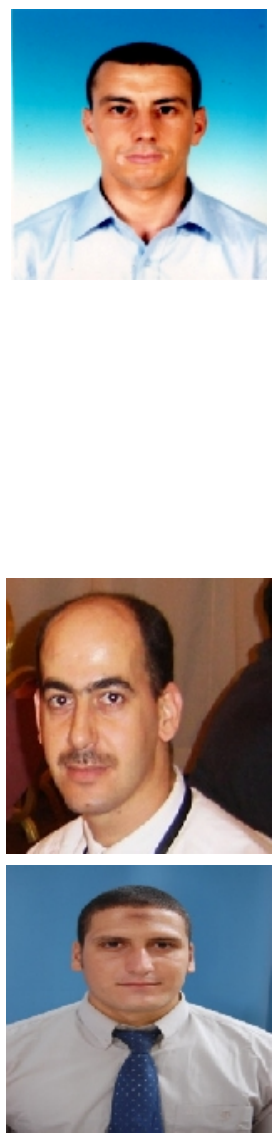